\title{
Latest results on high-energy cosmic neutrino searches with the ANTARES neutrino telescope
}

\author{
Agustín Sánchez Losa ${ }^{1, *}$ on behalf of the ANTARES Collaboration \\ ${ }^{1}$ INFN - Sezione di Bari, via Amendola 173, 70126 Bari, Italy
}

\begin{abstract}
The ANTARES detector is currently the largest undersea neutrino telescope. Located in the Mediterranean Sea at a depth of $2.5 \mathrm{~km}, 40 \mathrm{~km}$ off the Southern coast of France, it has been looking for cosmic neutrinos for more than 10 years. High-energy cosmic neutrino production is strongly linked with cosmic ray production. The latest results from IceCube Collaboration represent a step forward towards the confirmation of a highenergy cosmic ray source. The ANTARES location in the Northern Hemisphere is optimal for the observation of most of the Galactic Plane, including the Galactic Center. It has constrained the IceCube neutrino excess reports as well as, more recently, the flux from the source identified in the Blazar TXS 0506+056. The latest results of ANTARES on such analyses, including point-like and extended sources, diffuse fluxes, transient phenomena and multi-messenger studies, are presented..
\end{abstract}

\section{Introduction}

The ANTARES neutrino telescope [1] is located $40 \mathrm{~km}$ off the Southern coast of France, at a depth of $2.5 \mathrm{~km}$ in the Mediterranean Sea. It is currently the largest undersea neutrino telescope and has been looking for cosmic neutrinos for more than 10 years, with only a $20 \%$ efficiency loss on its optical modules [2].

Neutrino detection in neutrino telescopes is done via the Cherenkov light detection of the products of their interactions in the surroundings of the detector, in two main event categories or channels: tracks and showers.

Track-like events result from the reconstruction of a muon track, produced in charged current muon neutrino interactions and after some of the tau neutrino ones whenever the produced tau decays in a muon. Due to the long path of muons, track-like events is the channel with the largest statistics, yet cosmic ray induced down-going muons in the atmosphere limit its view range to the upgoing sky. Thanks to its location in the Northern Hemisphere and its latitude, ANTARES can see three-quarters of the sky using up-going track-like events, with an excellent view of most of the Galactic Plane, including the Galactic Center.

Shower-like events, when induced by a neutrino, come from the hadronic and electromagnetic showers originating within the detector, produced in neutral current interactions and in electron neutrino and sometimes tau neutrino charged current interactions. Due to its shorter range topology, many few showers than tracks are detected.

In both track and shower channels, an irreducible background of atmospheric neutrinos from the interaction of cosmic rays with the atmosphere prevents a certain cos-

*e-mail: agustin.sanchez@ba.infn.it mic origin identification on an event by event base, being necessary analyses with accumulative event statistics.

The ANTARES detector achieves an angular resolution below $0.4^{\circ}$ for tracks from neturinos with energies above $10 \mathrm{TeV}$ and a median angular resolution of the order of $3^{\circ}$ for showers [3]. The energy resolution on electron neutrino charged interactions is expected to be as low as $10 \%$ for neutrinos above $10 \mathrm{TeV}$ [4] (see figure 1).

High-energy cosmic neutrino production is strongly linked with cosmic ray origins. Results provided by IceCube, with measurements of a cosmic diffuse flux [5-8] and a strong correlation with a Blazar emission [9], start to prove this link. ANTARES, more sensitive at the lowest declinations and specially under the assumption of soft neutrino spectra, contributes with many different analyses, constraining independently the IceCube neutrino excess origin and the recent evidences of cosmic neutrino production in the Blazar TXS 0506+056. The latest analyses of ANTARES are presented, comprising diffuse flux, search for point-like source, multi-messenger and transient phenomena ones among others.

\section{Cosmic neutrinos from TXS 0506+056}

In [9] IceCube presented an analysis search for a neutrino emission excess from TXS 0506+056 with more than 9 years of data after a $290 \mathrm{TeV}$ neutrino induced muon track, IC170922A, was detected in the source direction during a gamma ray flare emission of the Blazar. The analysis found a $3.5 \sigma$ evidence of a transient neutrino emission on TXS 0506+056 direction between September 2014 and March 2015. ANTARES performed three analyses [10] looking for possible neutrino candidates compatible with TXS 0506+056 and the IceCube neutrino flares. 

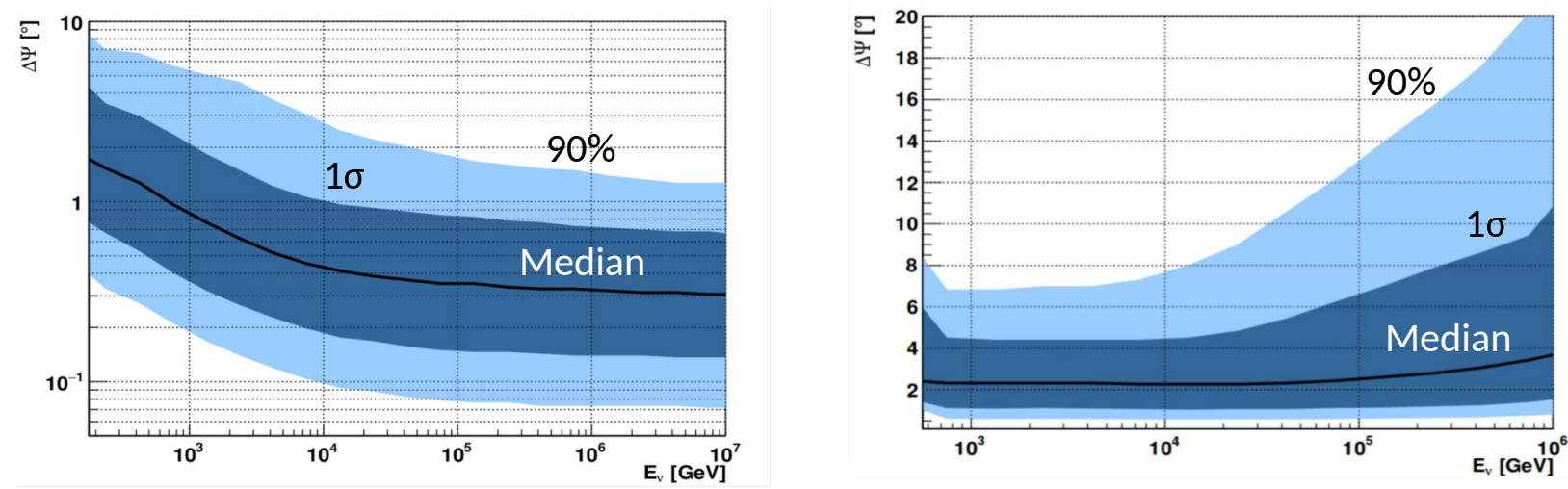

Figure 1. Median of the angle between the neutrino and the reconstructed muon track (left) and induced shower (right) directions for ANTARES for different neutrino energies [3, 4].

Neutrinos associated to the IceCube alert IC170922A were searched among the up-going high-energy neutrino candidates in the ANTARES online data stream reconstructed events [11] detected during the alert. These events are reconstructed with a fast algorithm, where a median angular resolution below $0.5^{\circ}$ is achieved for neutrino energies above $10 \mathrm{TeV}$. ANTARES has a competitive sensitivity with respect to IceCube in that sky region for $E_{v}<100 \mathrm{TeV}$. The search for up-going muon neutrinos in a $3^{\circ}$ cone around the IceCube alert in time windows of \pm 1 hour and \pm 1 day yielded negative results.

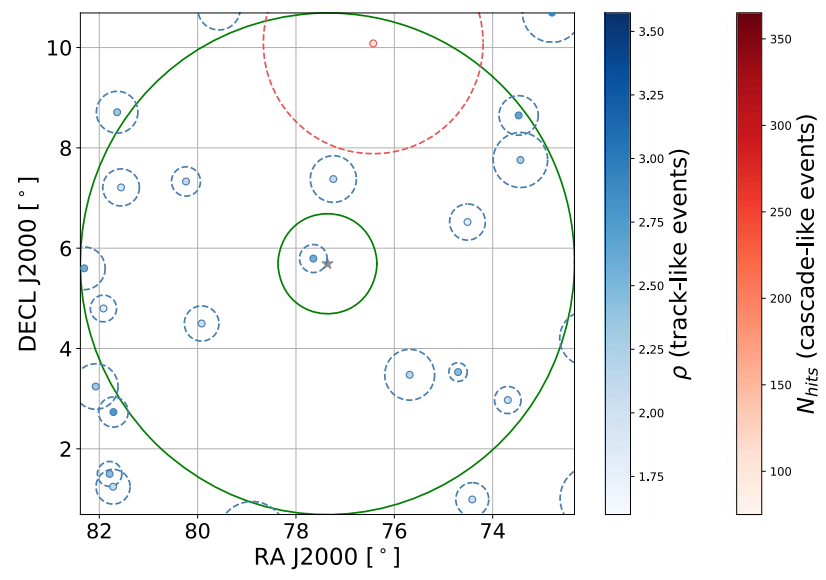

Figure 2. Selected ANTARES events from 2007-2017 around TXS 0506+056 (gray star) [10] with one and five degree radius green circles. Showers (red) and tracks (blue) shown dashed circles around them indicating the estimated angular error. Point color shades correspond to the energy estimators (legend on right).

A time integrated search of cosmic neutrinos from 107 candidate sources, including TXS 0506+056, was done with ANTARES data from 2007 to 2017, comprising 3136 days of livetime. This analysis is similar to the one presented in [12] (see section 3). In this search a pre-trial p-value of $3.4 \%$ (87\% post-trial) was found, with only two other candidate sources with a smaller p-value. The most significant event was a track at $0.3^{\circ}$ from the source on
December $12^{\text {th }}, 2013$ (see figure 2). An upper limit of $1.6 \times 10^{-8} \mathrm{GeV}^{-1} \mathrm{~cm}^{-2} \mathrm{~s}^{-1}$ in the $2 \mathrm{TeV}-4 \mathrm{PeV}$ range on the neutrino flux normalized at $100 \mathrm{TeV}$ was determined.

Finally it was realized a time-dependent search of neutrinos during the neutrino flares reported by IceCube in [9]. The ANTARES analysis is similar to those on [13, 14]. Two time profiles, concident to those most significative of IceCube, were analyzed: a flat neutrino emission from 56937.81 MJD to 57096.21 MJD and a Gaussian profile centered at $57004 \mathrm{MJD}$ and with $\sigma=55$ days. Despite of an event selection optimized to improve the sensitivity, no event during the described flares was found close to TXS 0506+056.

\section{Cosmic neutrino diffuse flux and point-like source searches}

ANTARES latest search for an isotropic cosmic neutrino flux [15] was performed on a data sample covering 9 years of operation of the detector with 2424 days of livetime from 2007 to 2015. Data selection, track and shower-like events, was done maximizing the analysis sensitivity while obtaining a high-purity neutrino sample, optimizing the event quality cuts and including this time a threshold on the energy estimators of tracks and showers.

The 33 unblinded events (see figure 3), 19 tracks plus 14 showers, provided a $1.6 \sigma$ excess, where the expected background from Monte Carlo simulations was of $24 \pm 7$ (13.5 tracks plus 10.5 showers) including statistical and systematic errors. These results, with the nosignal hypothesis excluded at $85 \%$ C.L. (see figure 4), are compatible with the IceCube flux. The simultaneous fit on the cosmic flux normalization and its spectral index, $\Gamma$, yielded a single-flavor normalization at $100 \mathrm{TeV}$ of $(1.7 \pm 1.0) \times 10^{-18} \mathrm{GeV}^{-1} \mathrm{~cm}^{-2} \mathrm{~s}^{-1} \mathrm{sr}^{-1}$ and $\Gamma=2.4_{-0.4}^{+0.5}$.

An analysis of the Fermi Bubbles region and the Galactic Ridge for a possible diffuse flux origin was also done [17], where the neutrinos in the studied on-zones were compared with the average from off-zones with equal extension and exposure. In this search, the expected diffuse galactic emission was estimated using the KRA $\gamma$ model, a radially dependent diffusion model for cosmic 

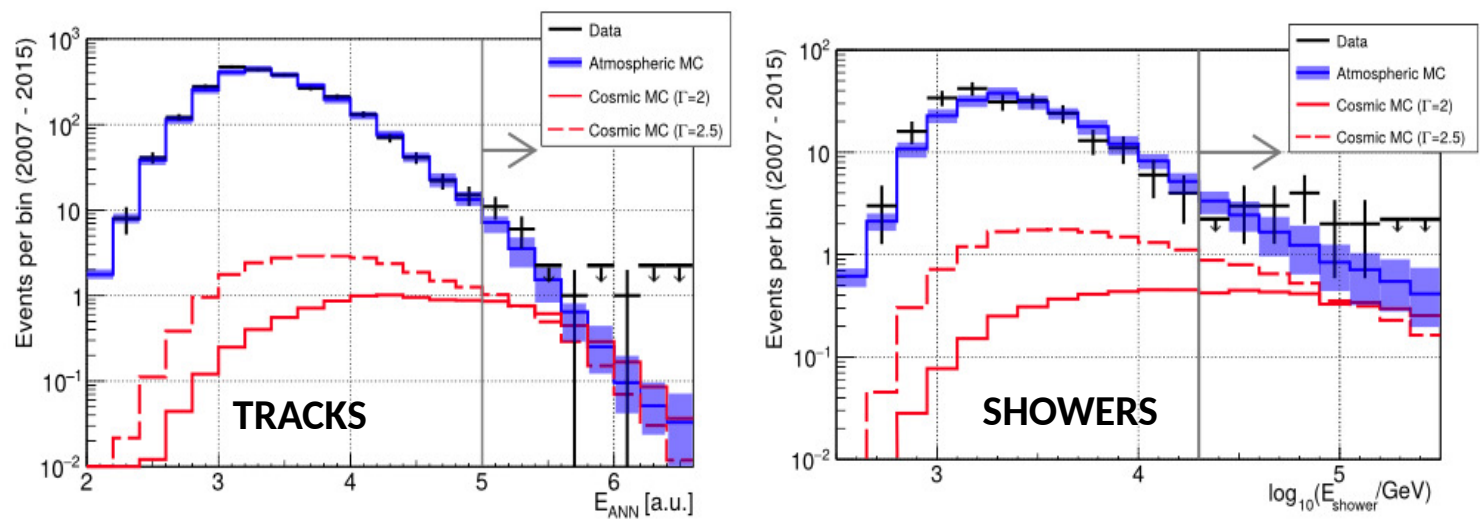

Figure 3. Track (left) and shower (right) energy estimator distributions for the latest ANTARES diffuse flux analysis [15]. Arrows mark the optimized energy estimator threshold for the analysis and estimations of the cosmic contribution for two spectral indexes, $\Gamma=2$ and $\Gamma=2.5$, are shown.

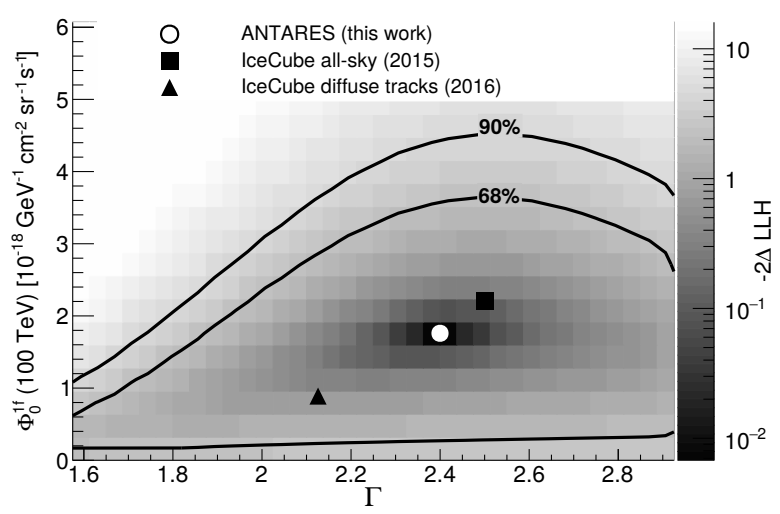

Figure 4. ANTARES 2D log-likelihood scan of the diffuse cosmic flux normalization and spectral index from [15]. Empty circle is the flux fit, showing also IceCube diffuse all-sky [16] and track $[7,8]$ analysis results and the $68 \%$ and $90 \%$ confidence contours. A $85 \%$ contour would start to exclude the null hypothesis.

rays in the Galaxy tuned on gamma-ray observations [18] and developed to explain the high-energy gamma ray diffuse Galactic emission observed by Fermi-LAT and dependent of the cosmic ray spectrum cut-off, where 5 and $50 \mathrm{PeV}$ values were considered. No significant excess was found in either regions, providing constrains (see figure 5) to the possible origin of the IceCube high-energy sample events (HESE).

In a recent combined analysis of ANTARES and IceCube of the Galactic Plane [19] it was even possible to start to constrain the KRA $\gamma$ model for a cosmic ray cut-off at $50 \mathrm{PeV}$ in the $0.09-300 \mathrm{TeV}$ range (see figure 6). This limits the total flux contribution of diffuse Galactic neutrino emission to the IceCube diffuse flux of [16] to less than $9 \%$ for neutrino energies above $30 \mathrm{TeV}$.

The latest ANTARES point source search [12] was performed with 2424 days of livetime from 2007 to 2015 , including 7622 tracks plus 180 showers. Four different searches were done on this data sample: a candidate list search of 106 objects (Blazars, supernova remnants, etc)

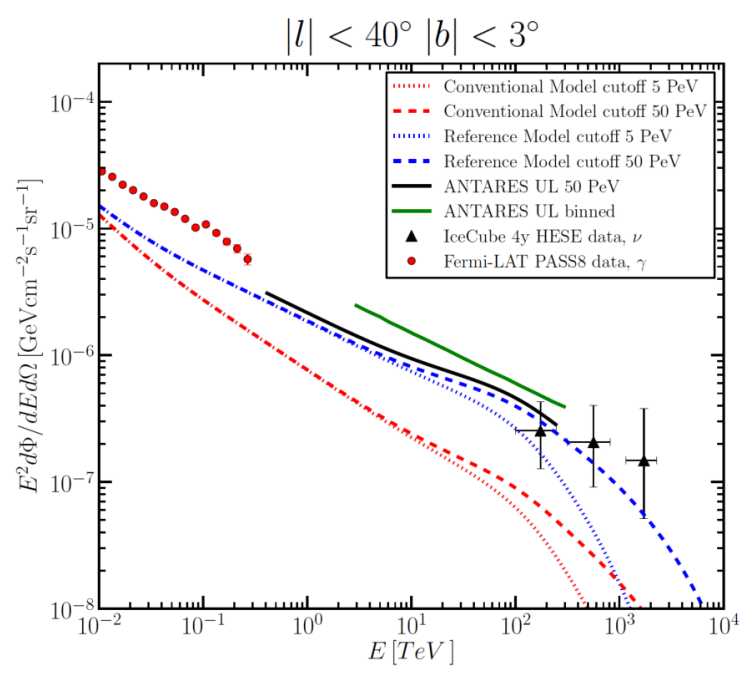

Figure 5. ANTARES upper limits on the three-flavor neutrino flux corresponding to the inner Galactic Plane region contained in $|l|<40^{\circ}$ and $|b|<3^{\circ}$ [17].

plus 13 IceCube HESE tracks; a full-sky scan for pointlike neutrino sources; an extended region conforming an ellipsoid $\left(15^{\circ} \times 20^{\circ}\right)$ around the Galactic Center, testing spectral indices 2.1, 2.3 and 2.5; and the Sagittarius $\mathrm{A}^{*}$ location with an extended Gaussian profile of widths $0^{\circ}$, $0.5^{\circ}, 1.0^{\circ}$ and $2.0^{\circ}$. No significant excess was found in any of the searches. HESS J0632+057 was the most significant result for the candidate list with a post-trial $\mathrm{p}$-value of $13 \%$. The most significant event cluster in the sky was at the $\left[\right.$ R.A. $\left.=343.8^{\circ}, \delta=23.5^{\circ}\right]$ coordinates with a $1.9 \sigma$ excess (p-value of 6\%). On figure 7, upper limits and sensitivities in function of the declination were compared with those of IceCube analyses [20, 21]: ANTARES can provide the best limits for a wide region of the Southern sky, specially for energies below $100 \mathrm{TeV}$. In order to exploit ANTARES advantage at lower energies and declinations together IceCube statistics and sensitivities at higher energies, there is an on-going combined analysis with 9 years 


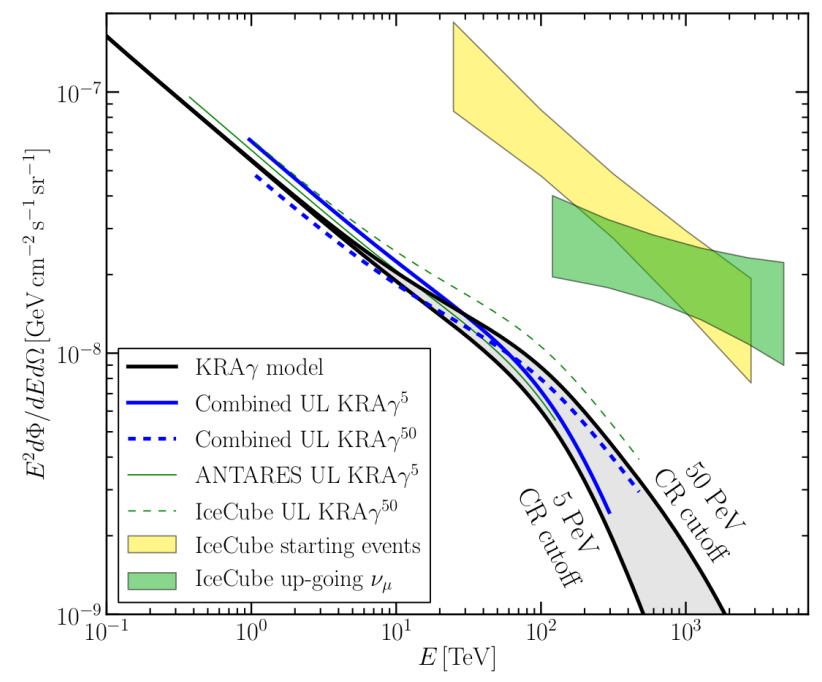

Figure 6. Combined ANTARES-IceCube upper limits [19] at 90\% C.L. (blue) on the three-flavor neutrino flux of the KRA $\gamma$ model, for 5 and $50 \mathrm{PeV}$ cutoff.

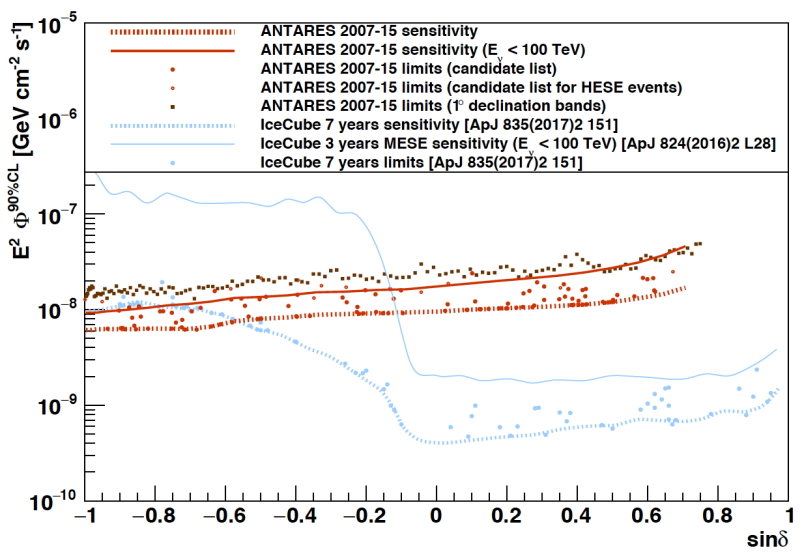

Figure 7. ANTARES $90 \%$ C.L. upper limits and sensitivities [12] for the studied candidate list as function of the declination for a $E_{\nu}^{-2}$ astrophysical neutrino flux with and without cutoff at $100 \mathrm{TeV}$ compared with IceCube equivalent results [20, 21]

of ANTARES data plus 7 years of IceCube samples for the Southern hemisphere.

\section{Multi-messenger and transient phenomena studies}

ANTARES has a strong commitment to the multimessenger approach, being part of many initiatives and programs where information is shared with multiple collaborations, comprising electromagnetic telescopes (radio, optical, X-rays and gamma-rays), other neutrino telescopes and cosmic ray and gravitational wave detectors. Transient sources are one of the most promising sources for the detection of cosmic neutrinos due to the reduced background they have as a consequence of search events in a well-defined space-time window. The studies carried by ANTARES can be summarized in three types: real-time follow-up analyses, alert triggering and off-line analyses.

\subsection{Real-time follow-up analyses}

ANTARES receives alerts of Gamma-Ray Bursts (GRBs), Fast Radio Bursts (FRBs) [22, 23], gravitational waves from LIGO and Virgo interferometers and even highenergy neutrino candidates from IceCube via the Gammaray Coordinated Network (GCN) or under private agreements of collaboration. ANTARES receives gravitational wave alerts in real time since the second observing run of Advanced LIGO and Virgo interferometers on November $30^{\text {th }}, 2016$. Real-time follow-up of the received alerts are performed searching for neutrino counterparts on ANTARES.

Since the ANTARES angular resolution (below $0.4^{\circ}$ above $10 \mathrm{TeV}$ ) is much smaller than the size of the gravitational wave error box (a few hundreds of square degrees on the sky) the size of the region of interest would be drastically reduced in case of a coincident neutrino detection. ANTARES together IceCube participated in the follow-up of the gravitational wave event GW150914, providing the first constraints on high-energy neutrino emission from a binary black hole coalescence [24]. Prompt searches of a neutrino counterpart to the first event involving a coalescence of two neutron stars (GW170817) were also performed with a subsequent refined study with the participation of ANTARES $[25,26]$. The neutrino non-detection in the source direction within a $\pm 500 \mathrm{~s}$ time window around the merger time is consistent with scenarios where the expected jet of highly energetic particles was produced off the line of sight of the source. Additional analyses of GW151226 [27] and GW170104 [28] were performed and recently was published the search of neutrino sources in ANTARES and IceCube in the first observing run of Advanced LIGO [29] where no significant coincident candidate was found, constraining the rate density of astrophysical sources dependent on their gravitational wave and neutrino emission processes.

\subsection{Alert triggering}

ANTARES also triggers electromagnetic follow-ups of neutrino events of potential astrophysical origin in the so-called TAToO (Telescopes-ANTARES Target-ofOpportunity) program [30], where optical telescopes (TAROT, MASTER, ZADKO), radio telescopes (MWA), X-ray satellites (Swift, Integral) and ground-based gamma-ray observatories (H.E.S.S., HAWC) perform a follow-up of the neutrino alerts ${ }^{1}$ [31]. Some characteristics of the triggers are high energetic events, doublets in a time window of 15 minutes or events which origin is compatible with a known close-by galaxy. The criteria for triggering specific instruments depend on the desired false alarm rate. Thanks to the very short alert-generation time of a few seconds and the half-sky simultaneous coverage, ANTARES is well-suited to detect transient sources. Since

\footnotetext{
${ }^{1}$ Example at http://www.astronomerstelegram.org/?read=7987
} 
TAToO beginning, in 2009, ANTARES has sent more than 300 alerts. The lack of observation of afterglows subsequent to the neutrino alerts disfavors strongly a GRB association to the neutrino event.

\subsection{Off-line analyses}

The significant identification of high-energy neutrinos in temporal and spatial coincidence with a variable electromagnetic known counterpart would unambiguously prove the existence of hadronic acceleration mechanisms in these sources. Off-line analyses search neutrino counterparts of cataloged flaring sources list (like Blazars [13] or microquasars) or also stacked event data-set, like those of GRBs, cosmic rays or even IceCube neutrino candidate events.

In a recent work, a search for neutrino emission during the flares of $34 \mathrm{X}$-ray binaries was performed by ANTARES [14]. The absence of correlation allowed to reject at $90 \%$ C.L. the most optimistic models for hadronic acceleration in these sources.

A search of neutrinos from four of the brightest GRBs visible by ANTARES between 2008 and 2013 (GRB080916C, GRB110918A, GRB130427A and GRB130505A) was also done [32] yielding to constrains of the internal shock and photospheric GRB model neutrino emissions.

The ANTARES data-set has been also scanned to look for time and space correlation with 54 IceCube events selected from two high-energy neutrino samples (the sample of charged current muon neutrinos from the Northern Hemisphere and the HESE sample) with the same analysis method as in [33] (paper of the updated work in preparation). Each IceCube event has been treated as a potential transient neutrino source. No ANTARES event was observed in correlation with the IceCube candidates which strongly constrains the possibility of transient origin of the IceCube events.

Finally, prior to this conference the latest ANTARES analysis correlation with cosmic rays was presented in [34], where 7629 tracks plus 180 showers of ANTARES from 2007 to 2015 were analyzed in the search of spatial correlation with 231 cosmic rays from Auger [35] plus 87 events from Telescope Array (TA), with no significant results. Currently a combined search of IceCube and ANTARES neutrino candidates in correlation with Auger and TA cosmic ray events is being carried out (see contribution [36] of this conference).

\section{Other analyses}

Additionally to these studies, ANTARES carry out a very complete program on indirect searches of dark matter, by looking for secondary particles produced when they decay or annihilate in very massive objects like the Earth [37], the Sun [38] or the Galactic Center [39]. In particular, WIMP (Weakly Interactive Massive Particles) candidates, that should accumulate in these objects due to scattering with the ordinary matter and the gravitation pull of these objects (since are expected to annihilate in pairs into a production of standard model particles, including neutrinos), should reveal as a neutrino excess in the direction of these sources. Considering its latitude, ANTARES has a better visibility of the Galactic Center and a low atmospheric background observation of the Sun than IceCube.

So far no excess has been observed on these analyses, providing of upper limits on these models (see figure 8). These limits are the most stringent for a certain region of the parameter space on the thermally averaged annihilation cross-section $\langle\sigma v\rangle$ for the $\tau^{+} \tau^{-}$channel in the dark matter search in the Galactic Center, while those on the spin-dependent (WIMP-proton) interaction cross section $\sigma_{S D}^{p}$ from the observations of the Sun, currently active neutrino telescopes produce the most stringent limits in the $M_{\text {WIMP }}>200 \mathrm{GeV}$ range, surpassing even directdetection experiments.

Since the ANTARES energy threshold is of a few tens of $\mathrm{GeV}$, it is possible to study the phenomenon of atmospheric muon neutrino disappearance due to neutrino oscillations and, in a similar way, to constrain the $3+1$ neutrino model, which foresees the existence of one sterile neutrino. A study with 10 years of ANTARES data (20072016) measured a $\Delta m_{32}^{2}=(2.0 \pm 0.3) \times 10^{-3} \mathrm{eV}^{2}$ and a $\theta_{23}=45^{\circ} \pm 12^{\circ}$ [40] (values consistent with world bestfits), excluded the non-oscillation hypothesis with a $4.6 \sigma$ significance and even yielded constrains on the $3+1$ neutrino model.

Finally, the pointing performance of the ANTARES detector was recently confirmed by a study of the Moon shadow effect [41], confirming the expected angular resolution of the telescope and its pointing accuracy.

\section{Conclusions}

ANTARES has recently turned 10 years old since its completion. During this time, ANTARES has provided to the scientific community numerous results on possible cosmic neutrino origins, including a wide multi-messenger live program, on neutrino nature and even on dark matter nature among others. With a median angular resolution below $0.4^{\circ}$ and a privileged position to observe the Galactic Center and most of the sky, ANTARES is the largest undersea neutrino telescope ever built and has contributed significantly to the dawn of the neutrino astronomy, settling also the path to the next generation of undersea neutrino telescopes: KM3NeT.

\section{References}

[1] M. Ageron et al., Nuclear Instruments and Methods in Physics Research Section A 656, 11 (2011)

[2] A. Albert et al., The European Physical Journal C 78, 669 (2018)

[3] S. Adrián-Martínez et al., The Astrophysical Journal Letters 786, L5 (2014)

[4] A. Albert et al., The European Physical Journal C 77, 419 (2017) 

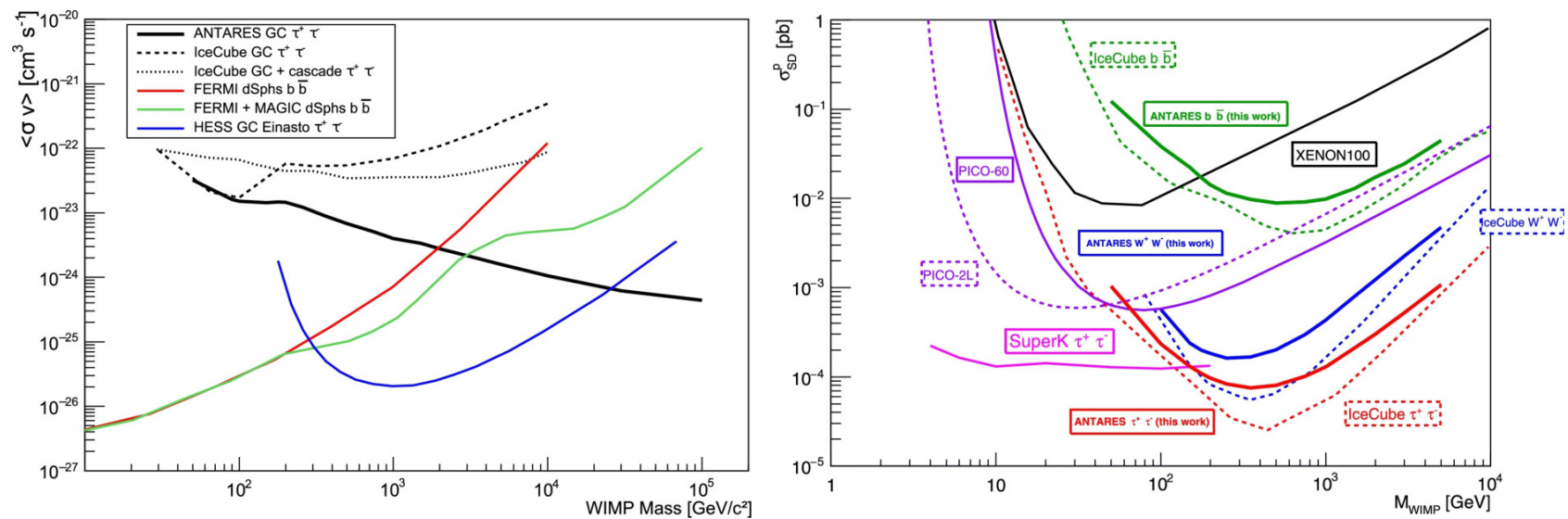

Figure 8. Left: ANTARES limits from the Milky Way on the thermally averaged annihilation cross-section, $\langle\sigma v\rangle$, as a function of the WIMP mass [39] compared to other experiment limits. Right: ANTARES limits on the spin-dependent WIMP-nucleon scattering cross-section as a function of WIMP mass for the $b \bar{b}, \tau^{+} \tau^{-}$and $W^{+} W^{-}$channels from observations of the Sun [38] in comparison to limits of other experiments.

[5] M. G. Aartsen et al., Physical Review Letters 113, 8 (2014)

[6] C. Kooper, Proceedings of Science 981 (ICRC2017)

[7] M. G. Aartsen et al., The Astrophysical Journal 833, 3 (2016)

[8] C. Haack, Proceedings of Science 1005 (ICRC2017)

[9] IceCube Collaboration, Science 361, 147-151 (2018)

[10] A. Albert et al., The Astrophysical Journal Letters 863, L30 (2018)

[11] M. Ageron et al., Astroparticle Physics 35, 530-536 (2012)

[12] A. Albert et al., Physical Review D 96, 082001 (2017)

[13] S. Adrián-Martínez et al., Journal of Cosmology and Astroparticle Physics 12, 014 (2015)

[14] A. Albert et al., Journal of Cosmology and Astroparticle Physics 04, 019 (2017)

[15] A. Albert et al., The Astrophysical Journal Letters 853, L7 (2018)

[16] M. G. Aartsen et al., The Astrophysical Journal 809, $98(2015)$

[17] A. Albert et al., Physical Review D 96, 062001 (2017)

[18] D. Gaggero et al., The Astrophysical Journal Letters 815, L25 (2015)

[19] A. Albert et al., The Astrophysical Journal Letters 868, L20 (2018)

[20] M. G. Aartsen et al., The Astrophysical Journal 835, 151 (2017)

[21] M. G. Aartsen et al., The Astrophysical Journal 824, L28 (2016)

[22] E. Petroff et al., Monthly Notices of the Royal Astronomical Society 469, 4465 (2017)

[23] S. Bhandari et al., Monthly Notices of the Royal Astronomical Society 475, 1427 (2018)
[24] S. Adrián-Martínez et al., Physical Review D 93, 122010 (2016)

[25] A. Albert et al., The Astrophysical Journal Letters 850, L35 (2017)

[26] B. P. Abbott et al., The Astrophysical Journal Letters 848, L12 (2017)

[27] A. Albert et al., Physical Review D 96, 022005 (2017)

[28] A. Albert et al., The European Physical Journal C 77, 911 (2017)

[29] A. Albert et al., The Astrophysical Journal 870, 2 (2019)

[30] S. Adrián-Martínez et al., Journal of Cosmology and Astroparticle Physics 02, 062 (2016)

[31] D. Dornic et al., Proceedings of Science 985 (ICRC2017)

[32] A. Albert et al., Monthly Notices of the Royal Astronomical Society 469, 906-915 (2017)

[33] J. Barrios et al., Proceedings of Science 987 (ICRC2017)

[34] J. Aublin et al., Proceedings of Science 990 (ICRC2017)

[35] A. Aab et al., The Astrophysical Journal 804, 15 (2015)

[36] L. Caccianiga, Web of Conferences 156 (UHECR2018)

[37] A. Albert et al., Physics of the Dark Universe 16, 41-48 (2017)

[38] S. Adrián-Martínez et al., Physics Letters B 759, 69-74 (2016)

[39] A. Albert et al., Physics Letters B 769, 249 (2017)

[40] A. Albert et al., arXiv:1812.08650 (Submitted to The Journal of High Energy Physics)

[41] A. Albert et al., The European Physical Journal C 78, 1006 (2018) 